\title{
The noncommutative infinitesimal equivariant index formula: part II
}

\author{
Yong Wang
}

\begin{abstract}
In this paper, we prove that infinitesimal equivariant Chern-Connes characters are well-defined. We decompose an equivariant index as a pairing of infinitesimal equivariant Chern-Connes characters with the Chern character of an idempotent matrix. We compute the limit of infinitesimal equivariant ChernConnes characters when the time goes to zero by using the Getzler symbol calculus and then extend these theorems to the family case. We also prove that infinitesimal equivariant eta cochains are well-defined and prove the noncommutative infinitesimal equivariant index formula for manifolds with boundary.
\end{abstract}

Keywords: Infinitesimal equivariant Chern-Connes characters; Getzler symbol calculus; infinitesimal equivariant eta cochains; infinitesimal equivariant family Chern-Connes characters.

MSC(2010): 58J20, 19K56

\section{Introduction}

The Atiyah-Bott-Segal-Singer index formula is a generalization of the Atiyah-Singer index theorem to manifolds admitting group actions. In [BV1], [LYZ], [PW], various heat kernel proofs of the equivariant index theorem have been given and each method has its own advantage. For manifolds with boundary, the equivariant extension of the Atiyah-Patodi-Singer index theorem was given by Donnelly in [Do]. In the equivariant Atiyah-Patodi-Singer index theorem, the equivariant eta invariant appears and the regularity of the equivariant eta invariant was proved by Zhang in [Zh]. An infinitesimal version of the equivariant index formula was established in [BV2] and a direct heat kernel proof was given by Bismut in [Bi]. The infinitesimal equivariant index formula for manifolds with boundary was established in [Go] with the introduction of the infinitesimal equivariant eta invariant.

The counterpart of the index formula in the noncommutative geometry is the computation of the Chern-Connes character [Co], [JLO], [GS]. The JLO character was computed in $[\mathrm{CM}]$ and $[\mathrm{BF}]$ by using the Getzler symbol calculus in [Ge2]. In $[\mathrm{Az}],[\mathrm{CH}]$ and $[\mathrm{PW}]$, these authors gave the computations of the equivariant JLO characters associated to a G-equivariant $\theta$-summable Fredholm module. In [Wa], we 
defined the truncated infinitesimal equivariant Chern-Connes characters and computed the limit of the truncated infinitesimal equivariant Chern-Connes characters when the time goes to zero.

Compared with [Wa], there are several improvements in the present paper. In (2.2) in [Wa], we defined truncated infinitesimal equivariant Chern-Connes characters. It is only well-defined when it is a polynomial of Lie algebra elements. In this paper, we drop off the truncated order $J$ (see (2.2)) and this consequently requires much better estimates (see Lemma 2.2). As in [GS], we decompose an equivariant index as a pairing of infinitesimal equivariant Chern-Connes characters with the Chern character of an idempotent matrix. Compared with Corollary 2.13 in [Wa], we drop off the limit on the right hand side of Corollary 2.13. Next we compute the limit of infinitesimal equivariant Chern-Connes characters when the time goes to zero by using the Getzler symbol calculus. Since we have dropped off the truncated order, (2.15) in [Wa] does not hold for our infinitesimal equivariant Chern-Connes characters. So we can not directly apply the method of Theorem 2.12 in [Wa]. Instead, we first apply the Getzler symbol calculus to prove the existence of the limit of infinitesimal equivariant Chern-Connes characters when time goes to zero (Theorem 3.9) and then use Theorem 2.12 in [Wa] to get the result. On the direction, in Section 3 in [Wa], we define the truncated infinitesimal equivariant eta cochains. Again in this paper we drop off the truncated order and then give a proof of the regularity at zero of infinitesimal equivariant eta cochains by using the method in $[\mathrm{PW}]$. That is, we prove that (3.5) in [Wa] holds for any $k$. This allows us to establish the noncommutative infinitesimal equivariant index formula for manifolds with boundary (see Theorem 4.9). In this paper, we also define family infinitesimal equivariant Chern-Connes characters and give the family generalization of the above theorems which does not appear in [Wa].

This paper is organized as follows: In Section 2, we prove that infinitesimal equivariant Chern-Connes characters are well-defined. Then we decompose the equivariant index as a pairing of infinitesimal equivariant Chern-Connes characters with the Chern character of an idempotent matrix. In Section 3, We compute the limit of infinitesimal equivariant Chern-Connes characters when the time goes to zero by using the Getzler symbol calculus. In Section 4, we prove that infinitesimal equivariant eta cochains are well-defined and prove the noncommutative infinitesimal equivariant index formula for manifolds with boundary. In Section 5, we extend results in Section 2,3 to the family case.

\section{The infinitesimal equivariant JLO cocycle and the in- dex pairing}

Let $M$ be a compact oriented even dimensional Riemannian manifold without boundary with a fixed spin structure and $S$ be the bundle of spinors on $M$. Denote by $D$ the associated Dirac operator on $H=L^{2}(M ; S)$, the Hilbert space of $L^{2}$-sections of the bundle $S$. Let $c(d f): S \rightarrow S$ denote the Clifford action with $f \in C^{\infty}(M)$. Suppose that $G$ is a compact connected Lie group acting on $M$ by orientation-preserving 
isometries preserving the spin structure and $\mathfrak{g}$ is the Lie algebra of $G$. Then $G$ commutes with the Dirac operator. For $X \in \mathfrak{g}$, let $X_{M}(p)=\left.\frac{d}{d t}\right|_{t=0} e^{-t X} p$ be the Killing field induced by $X$. Let $c(X)$ denote the Clifford action by $X_{M}$, and $\mathfrak{L}_{X}$ denote the Lie derivative respectively. Define $\mathfrak{g}$-equivariant modifications of $D$ and $D^{2}$ for $X \in \mathfrak{g}$ as follows:

$$
D_{X}:=D-\frac{1}{4} c(X) ; \quad H_{X}:=D_{-X}^{2}+\mathfrak{L}_{X}=\left(D+\frac{1}{4} c(X)\right)^{2}+\mathfrak{L}_{X} .
$$

Then $H_{X}$ is the equivariant Bismut Laplacian. Let $\mathbb{C}\left[\mathfrak{g}^{*}\right]$ denote the space of formal power series in $X \in \mathfrak{g}$ and $\psi_{t}$ be the rescaling operator on $\mathbb{C}\left[\mathfrak{g}^{*}\right]$ which is defined by $X \rightarrow \frac{X}{t}$ for $t>0$.

Let

$$
A=C_{G}^{\infty}(M)=\left\{f \in C^{\infty}(M) \mid f(g \cdot x)=f(x), g \in G, x \in M\right\},
$$

then the data $\left(A, H, D+\frac{1}{4} c(X), G\right)$ defines a non selfadjoint perturbation of finitely summable (hence $\theta$-summable) equivariant unbounded Fredholm module $(A, H, D, G)$ in the sense of $[\mathrm{KL}]$ (for details, see $[\mathrm{CH}]$ and $[\mathrm{KL}]$ ). For $\left(A, H, D+\frac{1}{4} c(X), G\right)$, the infinitesimal equivariant JLO cochain $\operatorname{ch}^{2 k}(D, X)$ can be defined by the formula:

$$
\begin{gathered}
\operatorname{ch}^{2 k}(D, X)\left(f^{0}, \cdots, f^{2 k}\right):=\int_{\triangle_{2 k}} \operatorname{Str}\left[e^{-\mathfrak{L}_{X}} f^{0} e^{-\sigma_{0}\left(D+\frac{1}{4} c(X)\right)^{2}} c\left(d f^{1}\right)\right. \\
\left.\cdot e^{-\sigma_{1}\left(D+\frac{1}{4} c(X)\right)^{2}} \cdots c\left(d f^{2 k}\right) e^{-\sigma_{2 k}\left(D+\frac{1}{4} c(X)\right)^{2}}\right] d \operatorname{Vol}_{\Delta_{2 k}},
\end{gathered}
$$

where $\triangle_{2 k}=\left\{\left(\sigma_{0}, \cdots, \sigma_{2 k}\right) \mid \sigma_{0}+\cdots \sigma_{2 k}=1\right\}$ is the $2 k$-simplex. For an integer $J \geq 0$, denote by $\mathbb{C}\left[\mathfrak{g}^{*}\right]_{J}$ the space of polynomials of degree $\leq J$ in $X \in \mathfrak{g}$ and let $(\cdot)_{J}: \mathbb{C}\left[\mathfrak{g}^{*}\right] \rightarrow \mathbb{C}\left[\mathfrak{g}^{*}\right]_{J}$ be the natural projection. Fix basis $e_{1}, \cdots, e_{n}$ of $\mathfrak{g}$ and let $X=x_{1} e_{1}+\cdots x_{n} e_{n}$. A $J$-degree polynomial on $X$ is namely a $J$-degree polynomial on $x_{1}, \cdots, x_{n}$. Now we prove that $\operatorname{ch}^{2 k}(D, X)\left(f^{0}, \cdots, f^{2 k}\right)$ is well-defined.

Let $H$ be a Hilbert space. For $q \geq 0$, denote by $\|.\|_{q}$ the Schatten $p$-norm on the Schatten ideal $L^{p}$. Let $L(H)$ denote the Banach algebra of bounded operators on $H$.

Lemma 2.1 ([Si]) (i) $\operatorname{Tr}(A B)=\operatorname{Tr}(B A)$, for $A, B \in L(H)$ and $A B, B A \in L^{1}$.

(ii) For $A \in L^{1}$, we have $|\operatorname{Tr}(A)| \leq\|A\|_{1},\|A\| \leq\|A\|_{1}$.

(iii) For $A \in L^{q}$ and $B \in L(H)$, we have: $\|A B\|_{q} \leq\|B\|\|A\|_{q},\|B A\|_{q} \leq\|B\|\|A\|_{q}$.

(iv) (Hölder Inequality) If $\frac{1}{r}=\frac{1}{p}+\frac{1}{q}, p, q, r>0, A \in L^{p}, B \in L^{q}$, then $A B \in L^{r}$ and $\|A B\|_{r} \leq\|A\|_{p}\|B\|_{q}$.

Let $H_{X}=D^{2}+F_{X}$, where $F_{X}$ is a first order differential operator with degree $\geq 1$ coefficients depending on $X$.

Lemma 2.2 For any $1 \geq u>0, t>0$, we have:

$\left\|e^{-u t H_{X}}\right\|_{u^{-1}} \leq 2 e^{\frac{t}{2}}\left\{1+\left[\left\|\left(1+D^{2}\right)^{-\frac{1}{2}} F_{X}\right\|^{2} e^{-1} \pi u t\right]^{\frac{1}{2}}\right\} e^{\left\|\left(1+D^{2}\right)^{-\frac{1}{2}} F_{X}\right\|^{2} e^{-1} \pi u t}\left(\operatorname{tr}\left[e^{-\frac{t D^{2}}{2}}\right]\right)^{u}$. 
Proof. By the Duhamel principle, it is that

$$
\begin{gathered}
\left\|e^{-u t H_{X}}\right\|_{u^{-1}}=\| \sum_{m \geq 0}(-u t)^{m} \int_{\triangle_{m}} e^{-v_{0} u t D^{2}} F_{X} e^{-v_{1} u t D^{2}} \\
\cdot F_{X} \cdots e^{-v_{m-1} u t D^{2}} F_{X} e^{-v_{m} u t D^{2}} d v \|_{u^{-1}}
\end{gathered}
$$

Also $\left\|(-u t)^{m} \int_{\triangle_{m}} e^{-v_{0} u t D^{2}} F_{X} e^{-v_{1} u t D^{2}} F_{X} \cdots e^{-v_{m-1} u t D^{2}} F_{X} e^{-v_{m} u t D^{2}} d v\right\|_{u^{-1}}$ is continuous and bounded by (2.7) in [Wa]. By the measure of the boundary of $\triangle_{m}$ being zero, we can estimate (2.4) in the interior of $\triangle_{m}$, that is $v_{j}>0$. It holds that

$$
\left\|e^{-\frac{v_{j}}{2} u t D^{2}} F_{X}\right\| \leq\left(v_{j} u t\right)^{-\frac{1}{2}} e^{-\frac{1-v_{j} u t}{2}}\left\|\left(1+D^{2}\right)^{-\frac{1}{2}} F_{X}\right\|,
$$

where we use that $F_{X}$ is a first order differential operator and the equality

$$
\sup \left\{(1+x)^{\frac{l}{2}} e^{-\frac{u t x}{2}}\right\}=(u t)^{-\frac{l}{2}} e^{-\frac{l-u t}{2}} .
$$

By the Hölder inequality, (2.4) and (2.5), the conditions that $0<u \leq 1$ and $v_{0}+$ $\cdots+v_{m-1} \leq 1$, we have

$$
\left\|e^{-u t H_{X}}\right\|_{u^{-1}} \leq e^{\frac{t}{2}} \sum_{m \geq 0} e^{-\frac{m}{2}}(u t)^{\frac{m}{2}}\left\|\left(1+D^{2}\right)^{-\frac{1}{2}} F_{X}\right\|^{m} \int_{\triangle_{m}} v_{0}^{-\frac{1}{2}} \cdots v_{m-1}^{-\frac{1}{2}} d v\left(\operatorname{tr}\left[e^{-\frac{t D^{2}}{2}}\right]\right)^{u} .
$$

It holds that (see line 7 in [BC, P.21])

$$
\int_{\triangle_{m}} v_{0}^{-\frac{1}{2}} \cdots v_{m-1}^{-\frac{1}{2}} d v=\frac{\pi^{\frac{m}{2}}}{\frac{m}{2} \Gamma\left(\frac{m+1}{2}\right)} .
$$

By $\Gamma(x+1)=x \Gamma(x), \Gamma(n)=(n-1) !$ and $\Gamma\left(\frac{1}{2}\right)=\sqrt{\pi}$, then $\Gamma\left(\frac{m+1}{2}\right)=\left(\frac{m-1}{2}\right)$ ! when $m$ is odd; $\Gamma\left(\frac{m+1}{2}\right)=\frac{(m-1) ! ! \sqrt{\pi}}{2^{\frac{m m}{2}}}$ when $m$ is even. By (2.8) and

$$
\lim _{m \rightarrow+\infty} \frac{(2 m-1) ! !}{(2 m) ! !}=0,
$$

we know that the series (2.7) is absolutely convergent. When $m$ is odd, then

$$
\frac{\pi^{\frac{m}{2}}}{\frac{m}{2} \Gamma\left(\frac{m+1}{2}\right)} \leq \frac{2 \pi^{\frac{m}{2}}}{\left(\frac{m+1}{2}\right) !}
$$

When $m$ is even, then

$$
\frac{\pi^{\frac{m}{2}}}{\frac{m}{2} \Gamma\left(\frac{m+1}{2}\right)} \leq \frac{2 \pi^{\frac{m}{2}}}{\left(\frac{m}{2}\right) !}
$$

By (2.7), (2.8), (2.10) and (2.11), we have

$$
\left\|e^{-u t H_{X}}\right\|_{u^{-1}} \leq 2 e^{\frac{t}{2}}\left[\sum_{m \text { even }} \frac{\left(\left\|\left(1+D^{2}\right)^{-\frac{1}{2}} F_{X}\right\|^{2} e^{-1} \pi u t\right)^{\frac{m}{2}}}{\left(\frac{m}{2}\right) !}\right.
$$




$$
\left.+\sum_{m \text { odd }} \frac{\left(\left\|\left(1+D^{2}\right)^{-\frac{1}{2}} F_{X}\right\|^{2} e^{-1} \pi u t\right)^{\frac{m}{2}}}{\left(\frac{m+1}{2}\right) !}\right]\left(\operatorname{tr}\left[e^{-\frac{t D^{2}}{2}}\right]\right)^{u} .
$$

Therefore, (2.3) can be obtained.

By $(2.2),(2.3)$ and the Hölder inequality as well as $\operatorname{Vol}_{\triangle_{2 k}}=\frac{1}{(2 k) !}$, for $t=1$ and $\sigma_{l} \leq 1$, we get

$$
\begin{gathered}
\left|\operatorname{ch}^{2 k}(D, X)\left(f^{0}, \cdots, f^{2 k}\right)\right| \leq \frac{1}{(2 k) !}\left\|f^{0}\right\|\left(\prod_{j=1}^{2 k}\left\|d f^{j}\right\|\right) \\
\cdot\left[2 e^{\frac{1}{2}}\left(1+\left(\left\|\left(1+D^{2}\right)^{-\frac{1}{2}} F_{X}\right\|^{2} e^{-1} \pi\right)^{\frac{1}{2}}\right)\right]^{2 k+1} e^{\left\|\left(1+D^{2}\right)^{-\frac{1}{2}} F_{X}\right\|^{2} e^{-1} \pi}\left(\operatorname{tr}\left[e^{-\frac{D^{2}}{2}}\right]\right) .
\end{gathered}
$$

Thus, $\operatorname{ch}^{2 k}(D, X)$ is well-defined. Recall that an even cochain $\left\{\Phi_{2 n}\right\}$ is called entire if $\sum_{n}\left\|\Phi_{2 n}\right\| n ! z^{n}$ is entire, where $\|\Phi\|:=\sup _{\left\|f^{j}\right\|_{1} \leq 1}\left\{\left|\Phi\left(f^{0}, f^{1}, \cdots, f^{2 k}\right)\right|\right\}$. By (2.13), then $\left\{\operatorname{ch}^{2 k}(D, X)\right\}$ is an entire cochain. Let $p \in M_{r}\left(\mathbb{C}^{\infty}(M)\right)$ and $p=p^{2}=p^{*}$ and $p(g x)=p(x)$. Define the Chern character of $p$ by (see $[\mathrm{GS}])$

$$
\operatorname{ch}(p):=\operatorname{Tr}(p)+\sum_{l}(-1)^{l} \frac{(2 l) !}{2 \cdot l !} \operatorname{Tr}(2 p-1, p, \cdots, p)_{2 l} .
$$

By $(2.13),\left\langle\operatorname{ch}^{*}(D, X), \operatorname{ch}(p)\right\rangle$ is convergent. Similarly to Theorem A in [GS], we have

Proposition 2.3 (1) The infinitesimal equivariant Chern-Connes character is closed:

$$
(B+b)\left(\operatorname{ch}^{*}(D, X)\right)=0 .
$$

(2) Let $D_{\tau}=D+\tau V$ and $D_{-X, \tau}=D_{-X}+\tau V$ and $V$ is a bounded operator which commutes with $e^{-X}$, then there exists a cochain $\operatorname{ch}^{*}\left(D_{\tau}, X, V\right)$ such that

$$
\frac{d}{d \tau} \operatorname{ch}^{*}\left(D_{\tau}, X\right)=-(B+b) \operatorname{ch}^{*}\left(D_{\tau}, X, V\right)
$$

By the Serre-Swan theorem, we denote the vector bundle over $M$ with the fibre $p(x)\left(\mathbb{C}^{r}\right)$ at $x \in M$ by $\operatorname{Im} p$. Let $D_{\operatorname{Im} p}$ be the Dirac operator twisted by the bundle $\operatorname{Im} p$. By Proposition 2.3, $(B+b) \operatorname{ch}(p)=0$ and Proposition 8.11 in [BGV], we have by taking $V=(2 p-1)[D, p]$ that (see Section 3 in $[\mathrm{GS}]$ )

Theorem 2.4 The following index formula holds

$$
\operatorname{Ind}_{e^{-X}}\left(D_{\operatorname{Im} p,+}\right)=\left\langle\operatorname{ch}^{*}(D, X), \operatorname{ch}(p)\right\rangle .
$$

In Theorem $2.4, X$ is unnecessarily small. 


\section{The computations of infinitesimal equivariant Chern- Connes characters}

In this section, we will compute infinitesimal equivariant Chern-Connes characters by Theorem 2.12 in [Wa] and the Getzler symbol calculus in [Ge2] and [BF]. Recall the Getzler symbol calculus in [Ge2] and [BF]. Let $E$ be a vector bundle over the compact manifold $M$ and $\pi: T^{*} M \rightarrow M$ be the natural map and $E^{0}=\pi^{*}(\operatorname{Hom}(E, E))$ be the pull-back of the bundle $\pi^{*}(\operatorname{Hom}(E, E))$ to a bundle over $T^{*} M$.

Definition 3.1 A section $p \in E^{0}$ is called a symbol of order $l$ if for every multi-index $\alpha$ and $\beta$ we have the estimates:

$$
\left\|\partial_{x}^{\alpha} \partial_{\xi}^{\beta} p(x, \xi)\right\| \leq C_{\alpha \beta}(1+|\xi|)^{m-|\beta|} .
$$

We denote by $\Sigma^{l}(E)$ the symbols of order $l$.

By the representative theorem of the Clifford algebra $C l\left(T^{*} M\right) \simeq \operatorname{Hom}(S(T M))$ and the isomorphism $C l\left(T^{*} M\right) \simeq \wedge\left(T^{*} M\right)$, note a map $\bar{\sigma}$ defined by

$$
\bar{\sigma}: \operatorname{Hom}(S(T M) \otimes E) \simeq \operatorname{Hom} E \otimes C l\left(T^{*} M\right) \simeq \operatorname{Hom} E \otimes \wedge\left(T^{*} M\right),
$$

and $\bar{\theta}$ is the inverse of $\bar{\sigma}$. Let $\mathbb{L}=\pi^{*}\left(\operatorname{Hom}(\mathrm{E}) \otimes \wedge\left(T^{*} M\right)\right) \otimes \mathbb{C}\left[\mathfrak{g}^{*}\right]$ and $X \in \mathfrak{g}$.

Definition 3.2 A section $p \in \mathbb{L}$ is called a $s$-symbol of order $l$ if

$$
p=\sum_{j=0}^{\operatorname{dim} M}\left(\sum_{|\alpha| \geq 0} p_{j, \alpha} X^{\alpha}\right) \otimes \omega_{j},
$$

where $\omega_{j} \in \Omega^{j}(M), p_{j, \alpha} \in \Sigma^{l-j-2|\alpha|}(E)$ and $\sum_{|\alpha| \geq 0}|| p_{j, \alpha}(x, \xi)||\left|X^{\alpha}\right|$ is convergent. We denote the collection of $s$-symbol of order $l$ by $S \Sigma^{l}(E, X)$.

Let $x_{0}$ be a fixed point in $M$ and $T_{x_{0}} M$ be the tangent space and exp be the exponential map respectively. Let $h$ be a function that is identically one in a neighborhood of the diagonal of $M \times M$ such that the exponential map is a diffeomorphism on the support of $h$. Let $\left(x_{0}, x\right) \in \operatorname{supp}(h)$. Let $\tau\left(x_{0}, x\right):(S(T M) \otimes E)_{x_{0}} \rightarrow(S(T M) \otimes E)_{x}$ be parallel translation about $\nabla^{S(T M) \otimes E}$ along the unique geodesic from $x_{0}$ to $x$. If $s \in \Gamma(S(T M) \otimes E)$, then we define

$$
\widehat{s}_{x_{0}}(x)=h\left(x_{0}, x\right) \tau\left(x, x_{0}\right) s(x) .
$$

We write $\widehat{s}_{x_{0}}(Y)$ instead of $\widehat{s}_{x_{0}}\left(\exp _{x_{0}} Y\right)$.

Let $\theta_{X}$ be the one-form associated with $X_{M}$ which is defined by $\theta_{X}(Y)=g(X, Y)$ for the vector field $Y$. Let $\nabla^{S, X}$ be the Clifford connection $\nabla^{S}-\frac{1}{4} \theta_{X}$ on the spinors

bundle and $\triangle_{X}$ be the Laplacian on $S(T M)$ associated with $\nabla^{S, X}$. Let $\mu(X)(\cdot)=$ 
$\nabla{ }^{T M} X_{M}$. Let $U=\left\{x \in T_{x_{0}} M\|\| x \|<\varepsilon\right\}$, where $\varepsilon$ is smaller than the injectivity radius of the manifold $M$ at $x_{0}$. Define $\alpha: U \times \mathfrak{g} \rightarrow \mathbb{C}$ via the formula

$$
\alpha_{X}(x):=-\frac{1}{4} \int_{0}^{1}\left(\iota(\mathcal{R}) \theta_{X}\right)(t x) t^{-1} d t, \quad \rho(X, x)=e^{\alpha_{X}(x)},
$$

where $\mathcal{R}=\sum_{i=1}^{n} x_{i} \frac{\partial}{\partial x_{i}}$. Then $\rho(X, 0)=1$. Recall ([BGV Lemma 8.13]) that the following identity holds

$$
H_{X}=-g^{i j}(x)\left(\nabla_{\partial_{i}}^{X} \nabla_{\partial_{j}}^{X}-\sum_{k} \Gamma_{i j}^{k} \nabla_{\partial_{k}}^{X}\right)+\frac{1}{4} r_{M},
$$

where $r_{M}$ is the scalar curvature and $\Gamma_{i j}^{k}$ is the connection coefficient of $\nabla^{L}$.

Let $\tau^{X}\left(x_{0}, x\right):(S(T M) \otimes E)_{x_{0}} \rightarrow(S(T M) \otimes E)_{x}$ be parallel translation about $\nabla^{S \otimes E, X}$ along the unique geodesic from $x_{0}$ to $x$. If $s \in \Gamma(S(T M) \otimes E)$, then we define

$$
\widehat{s}_{x_{0}}^{X}(x)=h\left(x_{0}, x\right) \tau^{X}\left(x, x_{0}\right) s(x) .
$$

Then

$$
\widehat{s}_{x_{0}}^{X}(x)=\rho \widehat{s}_{x_{0}}(x)
$$

where $\rho=\rho(X, x)$ is defined by $(3.5)$.

Definition 3.3 Let $p \in S \Sigma(E, X)$ and $s \in \Gamma(S(T M) \otimes E)$, then we define

$$
\theta(p)(s)\left(x_{0}\right)=\int_{T_{x_{0}} M \times T_{x_{0}}^{*} M} e^{-\sqrt{-1}\langle Y, \xi\rangle} \bar{\theta}(p)\left(x_{0}, \xi, X\right) \widehat{s}_{x_{0}}^{X}(Y) d Y d \xi .
$$

Remark. The operator $\theta(p)$ is well-defined since $\sum_{|\alpha| \geq 0}|| p_{j, \alpha}(x, \xi)||\left|X^{\alpha}\right|$ and $e^{\left|\alpha_{X}(x)\right|}$ are convergent. The operator $\theta(p)$ depends on the choice of the cut off function $h$, but the result does not depend on the cut off function for computations of infinitesimal equivariant Chern-Connes characters. We denote by $O p(E, X)$ all such operators with smoothing operators.

Definition 3.4 Given $s \in \Gamma(S(T M) \otimes E)$, define $\bar{s}_{x_{0}}^{X}(x)=h\left(x_{0}, x\right) \tau^{X}\left(x_{0}, x\right) s\left(x_{0}\right)$ and $\bar{s}_{x_{0}}(x)=h\left(x_{0}, x\right) \tau\left(x_{0}, x\right) s\left(x_{0}\right)$, then $\bar{s}_{x_{0}}^{X}(x)=\rho^{-1} \bar{s}_{x_{0}}(x)$. Let $P \in O p(E, X)$ and $s \in \Gamma(S(T M) \otimes E)$. Define $\sigma(P) \in \operatorname{End}(E)_{x_{0}} \otimes \Omega(M) \otimes \mathbb{C}\left[\mathfrak{g}^{*}\right]$ by

$$
\sigma(P)\left(x_{0}, \xi, X\right)=\left.\bar{\sigma} P_{y}\left(e^{\sqrt{-1}\left\langle\exp _{x_{0}}^{-1}(y), \xi\right\rangle} \bar{s}_{x_{0}}^{X}(y)\right)\right|_{y=x_{0}} .
$$

Lemma 3.5 Let $P=\sum_{\alpha} P_{\alpha} X^{\alpha} \in O p(E, X)$. If $\sum_{\alpha}|| P_{\alpha} \|_{1}\left|X^{\alpha}\right|$ is convergent, then $\sigma(P)$ is convergent. 
Proof. Since $\sum_{\alpha}|| P_{\alpha}\left|\|_{1}\right| X^{\alpha} \mid$ and $e^{\left|\alpha_{X}(x)\right|}$ are convergent, this comes from the definition 3.4 and $\left|e^{\sqrt{-1}\left\langle\exp _{x_{0}}^{-1}(y), \xi\right\rangle}\right|=1$ and $\left|h\left(x_{0}, x\right)\right| \leq 1$ and $\tau\left(x_{0}, x\right)$ being an isometry.

Lemma 3.6 Let $Y=\sum c_{i} \partial_{i}, Z=\sum d_{j} \partial_{j}$ with $c_{i}, d_{j} \in \mathbb{R}$. we have

$$
\begin{gathered}
\sigma\left(\nabla_{Y}^{X}\right)(x, \xi)=\sqrt{-1}\langle Y, \xi\rangle_{x} \\
\sigma\left(\nabla_{Y}^{X} \nabla_{Z}^{X}\right)(x, \xi)=-\langle Y, \xi\rangle\langle Z, \xi\rangle+\frac{1}{4}\left\langle R^{L}(Y, Z) \partial_{k}, \partial_{l}\right\rangle f^{k} \wedge f^{l}+\frac{1}{4}\left\langle\mu^{X}(Y), Z\right\rangle,
\end{gathered}
$$

where $f^{k}$ is the dual base of $\partial_{k}$.

Proof. By the definition 3.4, We have

$$
\sigma\left(\nabla_{Y}^{X}\right)\left(x_{0}, \xi\right)=\left.\bar{\sigma}\left[\nabla_{Y}^{X}\left(e^{\sqrt{-1}\left\langle\exp _{x_{0}}^{-1}(y), \xi\right\rangle} \rho^{-1} \bar{s}_{x_{0}}(y)\right)\right]\right|_{y=x_{0}}
$$

By

$$
\left.\left(d-\frac{1}{4} \theta_{X}\right)_{\partial_{j}}\left(\rho^{-1}\right)\right|_{x=x_{0}}=0 ;\left.\quad \nabla_{Y}\left(\bar{s}_{x_{0}}(x)\right)\right|_{x=x_{0}}=0,
$$

similarly to the computations of Example 1 in [BF], we get (3.11). We know that $\rho \nabla_{Y}^{X} \nabla_{Z}^{X} \rho^{-1}=\rho \nabla_{Y}^{X} \rho^{-1} \rho \nabla_{Z}^{X} \rho^{-1}$. By the appendix II in [ABP], we have

$$
\left.\nabla_{Y} \nabla_{Z} \bar{s}_{x_{0}}(y)\right|_{y=x_{0}}=\frac{1}{4}\left\langle R^{L}(Y, Z) \partial_{k}, \partial_{l}\right\rangle f^{k} \wedge f^{l} s\left(x_{0}\right) .
$$

In the trivialization of $S(T M)$, the conjugate $\rho(X, x)\left(\nabla_{\partial_{i}}^{S, X}\right) \rho(X, x)^{-1}$ is given by Lemma 8.13 in $[\mathrm{BGV}]$ which is

$$
\begin{gathered}
\rho(X, x)\left(\nabla_{\partial_{i}}^{S, X}\right) \rho(X, x)^{-1}=\partial_{i}+\frac{1}{4} \sum_{j, a<b}\left\langle R\left(\partial_{i}, \partial_{j}\right) e_{a}, e_{b}\right\rangle c\left(e_{a}\right) c\left(e_{b}\right) x^{j}-\frac{1}{4} \mu_{i j}^{M}(X) x^{j} \\
+\sum_{j<k} f_{i j k}(x) c\left(e_{j}\right) c\left(e_{k}\right)+g_{i}(x)+\left\langle h_{i}(x), X\right\rangle
\end{gathered}
$$

where $f_{i j k}(x)=O\left(|x|^{2}\right), g_{i}(x)=O(|x|)$, and $h_{i}(x)=O\left(|x|^{2}\right)$. By (3.15) and (3.16), similarly to the computations of Example 2 in $[\mathrm{BF}]$, we have (3.12).

Proposition 3.7 The following equality holds

$$
\sigma\left(H_{X}\right)=|\xi|^{2}+\frac{1}{4} r_{M}
$$

The operator $t^{2} H_{X}$ is an asymptotic pseudodifferential operator (see Definition 3.5 in $[B F])$.

Proof. By Lemma 3.6 and (3.6) and $g^{i j}\left(x_{0}\right)=\delta^{i j}, \Gamma_{i j}^{k}\left(x_{0}\right)=0$ and $R^{L}(Y, Y)=$ $\left\langle\mu^{X}(Y), Y\right\rangle=0$, we get Proposition 3.7. 
Definition 3.8 If $p(x, \xi, X) \in S \Sigma(E, X)$, then

$$
p_{t}(x, \xi, X)=\sum_{j=0}^{\operatorname{dim} M}\left(\sum_{|\alpha| \geq 0} p_{j, \alpha}(x, t \xi) t^{2|\alpha|} X^{\alpha}\right) \otimes \omega_{j} t^{j}
$$

Let $\psi_{t}: X \rightarrow \frac{X}{t}$ be the rescaling operator on the Lie algebra.

Theorem 3.9 For $P=\sum P_{\alpha} X^{\alpha} \in O P\left(S \Sigma^{-\infty}(E, X)\right)$ and $t>0$, then

$$
\psi_{t}^{2} \operatorname{Tr}_{\mathrm{S}}(P)=(2 \pi)^{-n}\left(\frac{2}{\sqrt{-1}}\right)^{\frac{n}{2}} \int_{M} \int_{T_{x_{0}}^{*} M} \operatorname{Tr}_{\mathrm{S}} \sigma(P)_{\frac{1}{t}}\left(x_{0}, \xi\right) d \xi d x .
$$

If $P=P_{t}$ and $P_{t}$ is an asymptotic pseudodifferential operator and $\sigma\left(P_{t}\right)(x, \xi)$ tends to zero when $|\xi|$ tends to infinity, then

$$
\psi_{t}^{2} \operatorname{Tr}_{\mathrm{s}}\left(P_{t}\right)=b_{0}+O(t)
$$

where $b_{0}$ is a constant.

Proof. By Theorem 3.7 in [Ge2], we have for any $s>0$ that

$$
\operatorname{Tr}_{\mathrm{S}}\left(P_{\alpha}\right)=(2 \pi)^{-n}\left(\frac{2}{\sqrt{-1}}\right)^{\frac{n}{2}} \int_{M} \int_{T_{x_{0}}^{*} M} \operatorname{Tr}_{\mathrm{s}} \sigma_{G}\left(P_{\alpha}\right)_{s}\left(x_{0}, \xi\right) d \xi d x
$$

where

$$
\sigma_{G}(P)\left(x_{0}, \xi, X\right)=\left.\bar{\sigma} P_{y}\left(e^{\sqrt{-1}\left\langle\exp _{x_{0}}^{-1}(y), \xi\right\rangle} \bar{s}_{x_{0}}(y)\right)\right|_{y=x_{0}} .
$$

Since $\sigma\left(P_{t}\right)(x, \xi)$ tends to zero when $|\xi|$ tends to infinity, by using the equality which will be proved in the following lemma 3.11

$$
\int_{T_{x_{0}}^{*} M} \operatorname{Tr}_{\mathrm{s}} \sigma_{G}(P)_{s}\left(x_{0}, \xi\right) d \xi d x=\int_{T_{x_{0}}^{*} M} \operatorname{Tr}_{\mathrm{s}} \sigma_{G}\left(\rho P \rho^{-1}\right)_{s}\left(x_{0}, \xi\right) d \xi d x,
$$

we have for $\rho\left(x_{0}\right)=1$ that

$$
\begin{aligned}
\int_{T_{x_{0}}^{*} M} \operatorname{Tr}_{\mathrm{s}} \sigma_{G}\left(P_{\alpha}\right)_{s}\left(x_{0}, \xi\right) d \xi & =\int_{T_{x_{0}}^{*} M} \operatorname{Tr}_{\mathrm{s}} \sigma_{G}\left(P_{\alpha} \rho^{-1}\right)_{s}\left(x_{0}, \xi\right) d \xi \\
& =\int_{T_{x_{0}}^{*} M} \operatorname{Tr}_{\mathrm{s}} \sigma\left(P_{\alpha}\right)_{s}\left(x_{0}, \xi\right) d \xi
\end{aligned}
$$

So

$$
\operatorname{Tr}_{\mathrm{S}}\left(P_{\alpha} X^{\alpha}\right)=(2 \pi)^{-n}\left(\frac{2}{\sqrt{-1}}\right)^{\frac{n}{2}} \int_{M} \int_{T_{x_{0}}^{*} M} \operatorname{Tr}_{\mathrm{s}} \sigma\left(P_{\alpha} \frac{X^{\alpha}}{s^{2|\alpha|}}\right)_{s}\left(x_{0}, \xi\right) d \xi d x
$$


Let $s=\frac{1}{t}$, then

$$
\operatorname{Tr}_{\mathrm{S}}\left(P_{\alpha} X^{\alpha}\right)=(2 \pi)^{-n}\left(\frac{2}{\sqrt{-1}}\right)^{\frac{n}{2}} \int_{M} \int_{T_{x_{0}}^{*} M} \operatorname{Tr}_{\mathrm{S}} \sigma\left(P_{\alpha} X^{\alpha} t^{2|\alpha|}\right)_{\frac{1}{t}}\left(x_{0}, \xi\right) d \xi d x .
$$

So

$$
\psi_{t}^{2} \operatorname{Tr}_{\mathrm{S}}\left(P_{\alpha} X^{\alpha}\right)=(2 \pi)^{-n}\left(\frac{2}{\sqrt{-1}}\right)^{\frac{n}{2}} \int_{M} \int_{T_{x_{0}}^{*} M} \operatorname{Tr}_{\mathrm{S}} \sigma\left(P_{\alpha} X^{\alpha}\right)_{\frac{1}{t}}\left(x_{0}, \xi\right) d \xi d x
$$

By taking the sum $\sum_{\alpha}$, we get (3.19). By Definitions 3.8 and Definition 3.5 in [BF], for the asymptotic pseudodifferential operator $P_{t}$, we have

$$
\sigma\left(P_{t}\right)=\sum_{l=0}^{+\infty} t^{l} p_{l}(x, \xi, X)_{t}
$$

so

$$
\sigma\left(P_{t}\right)_{\frac{1}{t}}=\sum_{l=0}^{+\infty} t^{l} p_{l}(x, \xi, X)
$$

By (3.19) and (3.29), we get (3.20).

Let $\mu^{M}$ be the Riemannian moment of $X$ defined by $\mu^{M}(X) Y=-\nabla_{Y} X^{M}$. Let $F_{\mathfrak{g}}^{M}(X)=\mu^{M}+R$ be the equivariant Riemannian curvature of $M$. The equivariant $\widehat{A}$-genus of the tangent bundle of $M$ is defined by $\widehat{A}\left(F_{\mathfrak{g}}^{M}(X)\right)=\operatorname{det}\left(\frac{F_{\mathfrak{g}}^{M}(X) / 2}{\sinh \left(F_{\mathfrak{g}}^{M}(X) / 2\right)}\right)^{\frac{1}{2}}$.

Theorem 3.10 When $2 k \leq \operatorname{dim} M$ and $X$ is small which means that $\left\|X_{M}\right\|$ is sufficiently small, then for $f^{j} \in C_{G}^{\infty}(M)$,

$$
\begin{gathered}
\lim _{t \rightarrow 0} \psi_{t} \operatorname{ch}^{2 k}(\sqrt{t} D, X)\left(f^{0}, \cdots, f^{2 k}\right) \\
=\frac{1}{(2 k) !}(2 \pi \sqrt{-1})^{-n / 2} \int_{M} f^{0} \wedge d f^{1} \wedge \cdots \wedge d f^{2 k} \widehat{A}\left(F_{\mathfrak{g}}^{M}(X)\right) d \operatorname{Vol}_{M} .
\end{gathered}
$$

Proof. In Theorem 3.9, let $P_{t}=t^{2 k} f^{0} e^{-\sigma_{0} t^{2} H_{X}} c\left(d f^{1}\right) \cdots c\left(d f^{2 k}\right) e^{-\sigma_{2 k} t^{2} H_{X}}$, then by Proposition 3.7, similarly to Lemma 3.13 in [BF], we have $P_{t}$ is an asymptotic pseudodifferential operator. By (3.20) and taking the $J$-jet, we have

$$
\lim _{t \rightarrow 0} \psi_{t}^{2} \operatorname{Tr}_{\mathrm{s}}\left(P_{t}\right)_{J}=b_{0, J}
$$

By Theorem 2.12 in [Wa], we have

$$
\lim _{t \rightarrow 0} \psi_{t}^{2} \operatorname{Tr}_{\mathrm{S}}\left(P_{t}\right)_{J}=\frac{1}{(2 k) !}(2 \pi \sqrt{-1})^{-n / 2} \int_{M} f^{0} \wedge d f^{1} \wedge \cdots \wedge d f^{2 k} \widehat{A}\left(F_{\mathfrak{g}}^{M}(X)\right)_{J} d \operatorname{Vol}_{M}
$$


By (3.31) and (3.32) and when $J$ goes to infinity, we obtain

$$
b_{0}=\frac{1}{(2 k) !}(2 \pi \sqrt{-1})^{-n / 2} \int_{M} f^{0} \wedge d f^{1} \wedge \cdots \wedge d f^{2 k} \widehat{A}\left(F_{\mathfrak{g}}^{M}(X)\right) d \mathrm{Vol}_{M} .
$$

By (3.20) and (3.33), when $t$ goes to zero, we get (3.30).

Lemma 3.11 The equality (3.23) holds.

Proof. Considering the equalities (70) and (71) in $[\mathrm{Pf}]$ (Note that these formulas hold for any pseudodifferential operators defined by (3.22) and not only for asymptotic pseudodifferential operators), let $N=0$, then

$$
\sigma_{G}\left(P \rho^{-1}\right)(x, \xi)=\sigma_{G}(P)(x, \xi) \rho^{-1}+r_{0}(\xi) .
$$

where $r_{0}(\xi)$ is defined by

$$
r_{0}(\xi)=\frac{\sqrt{-1}}{(2 \pi)^{n}} \sum_{j=1}^{n} \int_{T_{x_{0}}^{*} M(y)} \int_{0}^{1} \frac{\partial}{\partial y_{j}} a(\xi+s y) d s \cdot y_{j}\left[\mathcal{F}\left(f^{\psi}\right)\right](y) d y,
$$

and the Fourier transform $\mathcal{F}$ and $f^{\psi}$ are defined by (7) and (8) in [Pf] respectively, $a$ is the symbol of $P$. By (0.2) in [Ge2], we have the leading symbol of $e^{-t^{2} D^{2}}$ is $e^{-t^{2}|\xi|^{2}}$. As in (2.4), using the Duhamel principle, we expanse the operator $P_{t}$ and the leading symbol of $P_{t}$ is the product of $e^{-t^{2}|\xi|^{2}}$ and a polynomial on $\xi$. Without loss of generality, we assume $a=e^{-|\xi|^{2}}$. The following two well-known theorems are necessary:

I. Let $f(x, y)$ be continues on the domain $x \geq a, y \geq b$ and $\int_{b}^{+\infty} f(x, y) d y$ be uniformly convergent about $x$ on any finite interval included in $[a,+\infty]$ and $\int_{a}^{+\infty} f(x, y) d x$ be uniformly convergent about $y$ on any finite interval included in $[b,+\infty]$. We assume that the integral $\int_{b}^{+\infty}\left[\int_{a}^{+\infty}|f(x, y)| d x\right] d y$ or $\int_{a}^{+\infty}\left[\int_{b}^{+\infty}|f(x, y)| d y\right] d x$ exists, then

$$
\int_{a}^{+\infty}\left[\int_{b}^{+\infty} f(x, y) d y\right] d x=\int_{b}^{+\infty}\left[\int_{a}^{+\infty} f(x, y) d x\right] d y=\text { finite number. }
$$

II. There exists $\beta>0$, such that $|f(x, y)| \leq F(x)$ for any $x>\beta$ and $y \in I$ and that $\int_{a}^{+\infty} F(x) d x$ exists, then $\int_{a}^{+\infty} f(x, y) d x$ is uniformly convergent.

By (3.35), we consider

$$
\int_{T_{x_{0}}^{*} M(\xi)} r_{0}(\xi) d \xi=\left.\frac{\sqrt{-1}}{(2 \pi)^{n}} \sum_{j=1}^{n} \int_{T_{x_{0}}^{*} M(\xi)} \int_{T_{x_{0}}^{*} M(y)} \int_{0}^{1} \frac{\partial a}{\partial \xi_{j}}\right|_{\xi+s y} s d s \cdot y_{j}\left[\mathcal{F}\left(f^{\psi}\right)\right](y) d y d \xi .
$$


Since the Schwartz function $\left[\mathcal{F}\left(f^{\psi}\right)\right](y)$ is integral on $T_{x_{0}}^{*} M(y)$, we take some estimates on the right hand side of (3.37) in the polar coordinates of $T_{x_{0}}^{*} M(\xi)$ and $T_{x_{0}}^{*} M(y)$ and then we can verify that the right hand side of (3.37) satisfies the conditions of Theorem I. Using $\int_{T_{x_{0}}^{*} M(\xi)} \frac{\partial}{\partial \xi_{j}}\left[e^{-|\xi|^{2}} \xi^{\beta}\right] d \xi=0$ and (3.37), we get $\int_{T_{x_{0}}^{*} M} r_{0}(\xi)=0$. Therefore we get $(3.23)$.

Let

$$
\operatorname{Ch}(\operatorname{Im}(p))=\sum_{k=0}^{\infty}\left(-\frac{1}{2 \pi \sqrt{-1}}\right)^{k} \frac{1}{k !} \operatorname{Tr}\left[p(d p)^{2 k}\right] .
$$

We have

Corollary 3.12 When $X$ is small, then

$$
\operatorname{Ind}_{e^{-X}}\left(D_{\operatorname{Im} p,+}\right)=(2 \pi \sqrt{-1})^{-n / 2} \int_{M} \widehat{A}\left(F_{\mathfrak{g}}^{M}(X)\right) \operatorname{Ch}(\operatorname{Im} p) .
$$

Proof. Using the same discussions as those in [GS], we have the homotopy property of $\operatorname{ch}^{*}(D, X)$ for $t D_{-X}$. So by $(2.17)$, we have

$$
\operatorname{Ind}_{e^{-X}}\left(D_{\operatorname{Im} p,+}\right)=\left\langle\operatorname{ch}^{*}\left(t D_{-X}\right), \operatorname{ch}(p)\right\rangle,
$$

where

$$
\begin{gathered}
\operatorname{ch}^{2 k}\left(t D_{-X}\right)\left(f^{0}, \cdots, f^{2 k}\right):=t^{2 k} \int_{\triangle_{2 k}} \operatorname{Str}\left[e^{-\mathfrak{L}_{X}} f^{0} e^{-\sigma_{0} t^{2}\left(D+\frac{1}{4} c(X)\right)^{2}} c\left(d f^{1}\right)\right. \\
\left.\cdot e^{-\sigma_{1} t^{2}\left(D+\frac{1}{4} c(X)\right)^{2}} \cdots c\left(d f^{2 k}\right) e^{-\sigma_{2 k} t^{2}\left(D+\frac{1}{4} c(X)\right)^{2}}\right] d \operatorname{Vol}_{\Delta_{2 k}}
\end{gathered}
$$

In (3.40), let $e^{-X}=e^{-t^{2} X}$ and use $\left(\psi_{t}\right)^{2}$ acting on (3.40), then we get

$$
\operatorname{Ind}_{e^{-X}}\left(D_{\operatorname{Im} p,+}\right)=\left\langle\widetilde{\operatorname{ch}}^{*}(t D, X), \operatorname{ch}(p)\right\rangle,
$$

where

$$
\begin{gathered}
\widetilde{\operatorname{ch}}^{2 k}(t D, X)\left(f^{0}, \cdots, f^{2 k}\right):=t^{2 k} \int_{\triangle_{2 k}} \operatorname{Str}\left[f^{0} e^{-\sigma_{0} t^{2} H_{\frac{X}{t^{2}}}} c\left(d f^{1}\right)\right. \\
\left.\cdots c\left(d f^{2 k}\right) e^{-\sigma_{2 k} t^{2} H_{\frac{X}{t^{2}}}}\right] d \operatorname{Vol}_{\Delta_{2 k}} .
\end{gathered}
$$

Since $\operatorname{Ind}_{e^{-X}}\left(D_{\operatorname{Im} p,+}\right)$ is independent of $t$, taking the limit as $t \rightarrow 0$ in (3.42), we get by Theorem 3.10 that

$$
\operatorname{Ind}_{e^{-X}}\left(D_{\operatorname{Im} p,+}\right)=(2 \pi \sqrt{-1})^{-n / 2} \int_{M} \widehat{A}\left(F_{\mathfrak{g}}^{M}(X)\right) \operatorname{Ch}(\operatorname{Im} p) d \operatorname{Vol}_{M} .
$$




\section{The infinitesimal equivariant eta cochains}

In this Section, we prove the limit of truncated infinitesimal equivariant eta cochains exists when $J$ goes to infinity. By the Duhamel principle and (2.5), we have

$$
\begin{aligned}
& \left\|D_{-X} e^{-u t H_{X}}\right\|_{u^{-1}} \\
& \leq \sum_{m \geq 0}(u t)^{m} \int_{\triangle_{m}}\left\|D _ { - X } ( 1 + D ^ { 2 } ) ^ { - \frac { 1 } { 2 } } \left|\left\|||\left(1+D^{2}\right)^{\frac{1}{2}} e^{-\frac{\sigma_{0}}{2} u t D^{2}}\right\|\left\|e^{-\frac{\sigma_{0}}{2} u t D^{2}}\right\|_{\left(u \sigma_{0}\right)^{-1}}\right.\right. \\
& \cdot\left\|F _ { X } ( 1 + D ^ { 2 } ) ^ { - \frac { 1 } { 2 } } \left|\left\|||\left(1+D^{2}\right)^{\frac{1}{2}} e^{-\frac{\sigma_{1}}{2} u t D^{2}}\right\|\left\|e^{-\frac{\sigma_{1}}{2} u t D^{2}}\right\|_{\left(u \sigma_{1}\right)^{-1}}\right.\right. \\
& \cdots\left\|F_{X}\left(1+D^{2}\right)^{-\frac{1}{2}}\right\|\left\|\mid\left(1+D^{2}\right)^{\frac{1}{2}} e^{-\frac{\sigma_{m}}{2} u t D^{2}}\right\|\left\|e^{-\frac{\sigma_{m}}{2} u t D^{2}}\right\|_{\left(u \sigma_{m}\right)^{-1}} d \sigma \\
& \leq\left\|D_{-X}\left(1+D^{2}\right)^{-\frac{1}{2}}\right\|(\text { eut })^{-\frac{1}{2}} \sum_{m \geq 0}\left(e^{-1} u t\left\|F_{X}\left(1+D^{2}\right)^{-\frac{1}{2}}\right\|^{2}\right)^{\frac{m}{2}} \\
& \cdot e^{\frac{u t}{2}}\left(\operatorname{tr} e^{-\frac{t}{2} D^{2}}\right)^{u} \int_{\triangle_{m}} \sigma_{0}^{-\frac{1}{2}} \cdots \sigma_{m}^{-\frac{1}{2}} d \sigma \\
& \leq\left\|D_{-X}\left(1+D^{2}\right)^{-\frac{1}{2}}\right\|(u t)^{-\frac{1}{2}} 2 e^{\frac{u t}{2}}\left\{1+\left[\left\|F_{X}\left(1+D^{2}\right)^{-\frac{1}{2}}\right\|^{2} e^{-1} \pi u t\right]^{\frac{1}{2}}\right\} \\
& e^{\left\|F_{X}\left(1+D^{2}\right)^{-\frac{1}{2}}\right\|^{2} \pi u t}\left(\operatorname{tr} e^{-\frac{t}{2} D^{2}}\right)^{u},
\end{aligned}
$$

where

$$
\int_{\triangle_{m}} \sigma_{0}^{-\frac{1}{2}} \cdots \sigma_{m}^{-\frac{1}{2}} d \sigma=\frac{\pi^{\frac{m+1}{2}}}{\Gamma\left(\frac{m}{2}+1\right)}
$$

and

$$
\begin{aligned}
& \frac{\pi^{\frac{m+1}{2}}}{\Gamma\left(\frac{m}{2}+1\right)}=\frac{\pi^{\frac{m+1}{2}}}{\left(\frac{m}{2}\right) !}, \text { when } m \text { is even, } \\
& \frac{\pi^{\frac{m+1}{2}}}{\Gamma\left(\frac{m}{2}+1\right)} \leq \frac{2 \pi^{\frac{m}{2}}}{\left(\frac{m-1}{2}\right) !}, \quad \text { when } m \text { is odd. }
\end{aligned}
$$

Now let $M$ be a compact oriented odd dimensional Riemannian manifold without boundary with a fixed spin structure and $S$ be the bundle of spinors on $M$. The fundamental setup consists with that on page 2. Let $K_{t}=\sqrt{t}\left(D+\frac{c(X)}{4 t}\right)$, then $\frac{d K_{t}}{d t}=\frac{1}{2 \sqrt{t}} D_{\frac{X}{t}}$. For $a_{0}, \cdots, a_{2 k} \in C_{G}^{\infty}(M)$, we define the infinitesimal equivariant cochain $\operatorname{ch}_{X}^{2 k}\left(K_{t}, \frac{d K_{t}}{d t}\right)$ by the formula:

$$
\begin{gathered}
\operatorname{ch}_{X}^{2 k}\left(K_{t}, \frac{d K_{t}}{d t}\right)\left(a_{0}, \cdots, a_{2 k}\right) \\
=\sum_{j=0}^{2 k}(-1)^{j}\left\langle a_{0},\left[K_{t}, a_{1}\right], \cdots,\left[K_{t}, a_{j}\right], \frac{d K_{t}}{d t},\left[K_{t}, a_{j+1}\right], \cdots,\left[K_{t}, a_{2 k}\right]\right\rangle_{t}(X) .
\end{gathered}
$$

If $A_{j}(0 \leq j \leq q)$ are operators on $\Gamma(M, S(T M))$, we define

$$
\left\langle A_{0}, \cdots, A_{q}\right\rangle_{t}(X)=\int_{\triangle_{q}} \operatorname{tr}\left[e^{-L_{X}} A_{0} e^{-\sigma_{0} K_{t}^{2}} A_{1} e^{-\sigma_{1} K_{t}^{2}} \cdots A_{q} e^{-\sigma_{q} K_{t}^{2}}\right] d \sigma,
$$


where $\triangle_{q}=\left\{\left(\sigma_{0}, \cdots, \sigma_{q}\right) \mid \sigma_{0}+\cdots+\sigma_{q}=1, \sigma_{j} \geq 0\right\}$ is a simplex in $\mathbf{R}^{\mathbf{q}}$ and $L_{X}$ is the Lie derivative generated by $X$ on the spinors bundle.

Formally, the infinitesimal equivariant eta cochain for the odd dimensional manifold is defined to be an even cochain sequence by the formula:

$$
\eta_{X}^{2 k}(D)=\frac{1}{\sqrt{\pi}} \int_{0}^{\infty} \operatorname{ch}_{X}^{2 k}\left(K_{t}, \frac{d K_{t}}{d t}\right) d t
$$

Then $\eta_{X}^{0}(D)(1)$ is the half of the infinitesimal equivariant eta invariant defined by Goette in [Go]. In order to prove that the above expression is well defined, it is necessary to check the integrality near the two ends of the integration. Firstly, the regularity at infinity comes from the following lemma.

Lemma 4.1 For $a_{0}, \cdots, a_{2 k} \in C_{G}^{\infty}(M)$, we have

$$
\operatorname{ch}_{X}^{2 k}\left(K_{t}, \frac{d K_{t}}{d t}\right)\left(a_{0}, \cdots, a_{2 k}\right)=O\left(t^{-\frac{3}{2}}\right), \text { as } t \rightarrow \infty .
$$

Proof. Let $L_{0}$ be a fixed large number. Then $\frac{1}{\Gamma\left(\frac{1}{2}\right)} \int_{\varepsilon}^{L_{0}} \operatorname{ch}_{X}^{2 k}\left(K_{t}, \frac{d K_{t}}{d t}\right)\left(a_{0}, \cdots, a_{2 k}\right) d t$ is well-defined by Lemma 2.2 and (4.1). Similarly to Lemma 2.2 and (4.1), we know that Lemma 3.5 in [Wa] holds when $J$ goes to infinity. So $\frac{1}{\Gamma\left(\frac{1}{2}\right)} \int_{L_{0}}^{\infty} \operatorname{ch}_{X}^{2 k}\left(K_{t}, \frac{d K_{t}}{d t}\right) d t$ is well-defined and Lemma 4.1 holds.

Next, we prove the regularity at zero. Let $F_{*}=D_{-X}^{2}$ and $\widehat{F_{*}}=H_{X}-d t D_{X}$ where $d t$ is an auxiliary Grassmann variable as shown in $[\mathrm{BiF}]$. Then $t \psi_{t} \widehat{F_{*}}=t H_{\frac{X}{t}}-$ $2 t^{\frac{3}{2}} d t \frac{d K_{t}}{d t}$. Let

$$
\begin{gathered}
\operatorname{ch}^{2 k}\left(\widehat{F_{*}}\right)\left(a_{0}, \cdots, a_{2 k}\right)=t^{k} \int_{\triangle_{2 k}} \psi_{t} \operatorname{tr}\left[a_{0} e^{-t \sigma_{0} \widehat{F_{*}}}\left[D, a_{1}\right] \cdots\left[D, a_{2 k}\right] e^{-t \sigma_{2 k} \widehat{F_{*}}}\right] d \sigma, \\
\operatorname{ch}^{2 k}\left(F_{*}\right)\left(a_{0}, \cdots, a_{2 k}\right)=t^{k} \int_{\triangle_{2 k}} \psi_{t} \operatorname{tr}\left[a_{0} e^{-t \sigma_{0} H_{X}}\left[D, a_{1}\right] \cdots\left[D, a_{2 k}\right] e^{-t \sigma_{2 k} H_{X}}\right] d \sigma .
\end{gathered}
$$

By the Duhamel principle and $d t^{2}=0$, we have

$$
\begin{aligned}
e^{-t \sigma_{j} \psi_{t} \widehat{F_{*}}} & =e^{-t \sigma_{j} \psi_{t} H_{X}}+\int_{0}^{1} e^{-(1-a) t \sigma_{j} \psi_{t} H_{X}}\left(2 t^{\frac{3}{2}} d t \frac{d K_{t}}{d t}\right) e^{-a t \sigma_{j} \psi_{t} H_{X}} d\left(\sigma_{j} a\right) \\
& =e^{-t \sigma_{j} \psi_{t} H_{X}}+2 t^{\frac{3}{2}} d t \int_{0}^{\sigma_{j}} e^{-\left(\sigma_{j}-\xi\right) t \psi_{t} H_{X}} \frac{d K_{t}}{d t} e^{-t \xi \psi_{t} H_{X}} d \xi
\end{aligned}
$$

By (4.5) and (4.9)-(4.11) and $d t^{2}=0$, we get

$$
\operatorname{ch}^{2 k}\left(\widehat{F_{*}}\right)\left(a_{0}, \cdots, a_{2 k}\right)=\operatorname{ch}^{2 k}\left(F_{*}\right)\left(a_{0}, \cdots, a_{2 k}\right)-2 t^{\frac{3}{2}} \operatorname{ch}_{X}^{2 k}\left(K_{t}, \frac{d K_{t}}{d t}\right)\left(a_{0}, \cdots, a_{2 k}\right) d t .
$$


Lemma 4.2 The following estimate holds

$$
\operatorname{ch}_{X}^{2 k}\left(K_{t}, \frac{d K_{t}}{d t}\right) \sim O(1) \text { when } t \rightarrow 0
$$

Proof. By (4.12), we only need to prove

$$
\operatorname{ch}^{2 k}\left(\widehat{F_{*}}\right)\left(a_{0}, \cdots, a_{2 k}\right)-\operatorname{ch}^{2 k}\left(F_{*}\right)\left(a_{0}, \cdots, a_{2 k}\right)=O\left(t^{\frac{3}{2}}\right) d t .
$$

Let

$$
\begin{aligned}
Q_{\widehat{F_{*}}} & =a_{0}\left(\widehat{F_{*}}+\partial_{t}\right)^{-1} c\left(d a_{1}\right) \cdots c\left(d a_{2 q}\right)\left(\widehat{F_{*}}+\partial_{t}\right)^{-1}, \\
Q_{F_{*}} & =a_{0}\left(F_{*}+\partial_{t}\right)^{-1} c\left(d a_{1}\right) \cdots c\left(d a_{2 q}\right)\left(F_{*}+\partial_{t}\right)^{-1} .
\end{aligned}
$$

By using Lemma 8.4 in $[\mathrm{PW}]$, we have

$$
\begin{gathered}
t^{q} \psi_{t}\left[a_{0} e^{-t \sigma_{0} \widehat{F_{*}}}\left[D, a_{1}\right] \cdots\left[D, a_{2 q}\right] e^{-t \sigma_{2 q} \widehat{F_{*}}}\right](x, y)=t^{-q} \psi_{t} K_{Q_{\widehat{F_{*}}}}(x, y, t) ; \\
t^{q} \psi_{t}\left[a_{0} e^{-t \sigma_{0} H_{X}}\left[D, a_{1}\right] \cdots\left[D, a_{2 q}\right] e^{-t \sigma_{2 q} H_{X}}\right](x, y)=t^{-q} \psi_{t} K_{Q_{F_{*}}}(x, y, t) .
\end{gathered}
$$

So we only need to prove

$$
t^{-q} \psi_{t} \operatorname{tr}\left[K_{Q_{\widehat{F_{*}}}}(x, x, t)-K_{Q_{F_{*}}}(x, x, t)\right]=O\left(t^{\frac{3}{2}}\right) d t .
$$

By the trace property, we have

$$
\begin{gathered}
t^{-q} \psi_{t} \operatorname{tr}\left[K_{Q_{\widehat{F *}}}(x, x, t)-K_{Q_{F_{*}}}(x, x, t)\right] \\
=t^{-q} \psi_{t} \operatorname{tr}\left[K_{Q_{h \widehat{F}_{*}(h \rho)-1}}(x, x, t)-K_{Q_{\rho H_{X} \rho^{-1}}}(x, x, t)\right] .
\end{gathered}
$$

By (3.15), (3.18) and (3.24) in [Wa] and $d t^{2}=0$ where we use $d t$ instead of $z$ in [Wa], we have

$$
\begin{gathered}
t^{-q} \psi_{t}\left[Q_{h \rho \widehat{F_{*}}(h \rho)^{-1}}-Q_{\rho H_{X} \rho^{-1}}\right] \\
=-t^{-q} d t \psi_{t}\left[a_{0}\left(\partial_{t}+\rho H_{X} \rho^{-1}\right)^{-1} u\left(\partial_{t}+\rho H_{X} \rho^{-1}\right)^{-1}\right. \\
\cdot c\left(d a_{1}\right) \cdots c\left(d a_{2 q}\right)\left(\partial_{t}+\rho H_{X} \rho^{-1}\right)^{-1} \\
+\cdots+a_{0}\left(\partial_{t}+\rho H_{X} \rho^{-1}\right)^{-1} \cdots c\left(d a_{2 q}\right) \\
\left.\cdot\left(\partial_{t}+\rho H_{X} \rho^{-1}\right)^{-1} u\left(\partial_{t}+\rho H_{X} \rho^{-1}\right)^{-1}\right] .
\end{gathered}
$$

By $O_{G}(u) \leq 0$ and $O_{G}\left(\left(\partial_{t}+\rho H_{X} \rho^{-1}\right)^{-1}\right)=-2$, we have

$$
\begin{gathered}
O_{G}\left[\left(\partial_{t}+\rho H_{X} \rho^{-1}\right)^{-1} u\left(\partial_{t}+\rho H_{X} \rho^{-1}\right)^{-1}\right. \\
\left.\cdot c\left(d a_{1}\right) \cdots c\left(d a_{2 q}\right)\left(\partial_{t}+\rho H_{X} \rho^{-1}\right)^{-1}\right]=-2 q-4,
\end{gathered}
$$

which has odd Clifford elements. When we drop off the truncated order $J$ in Lemma 2.9 in [Wa] and consider the convergent series on $X$ as in the definition 3.2, we know 
that Lemma 2.9 in [Wa] holds for our operator in (4.22). By (4.20)-(4.22) and Lemma 2.91 ) in [Wa] for $j=n$ and $m=-2 q-4$, we get (4.19).

Remark. Similarly to Proposition 1.2 in $[\mathrm{Wu}]$, We use the symbol calculus about the connection $\nabla^{X}$ in Section 3 instead of the Getzler symbol calculus in Proposition $1.2 \mathrm{in}[\mathrm{Wu}]$, then we can give another proof of Lemma 4.2.

Again Proposition 3.8 in [Wa] holds, we have

Proposition 4.3 Assume that $D$ is invertible with $\lambda$ being the smallest positive eigenvalue of $|D|$ and $\|d p\|<\lambda$, then the pairing $\left\langle\eta_{X}^{*}(D), \mathrm{ch}_{*}(p)\right\rangle$ is well-defined.

We also have the following theorem.

Theorem 4.4 Assume $D$ is invertible and $\|d p\|<\lambda$ where $\lambda$ is the smallest eigenvalue of $|D|$, then we have

$$
\frac{1}{2} \eta_{X}\left(p\left(D \otimes I_{r}\right) p\right)=\left\langle\eta_{X}^{*}(D), \operatorname{ch}_{*}(p)\right\rangle
$$

where $\eta_{X}\left(p\left(D \otimes I_{r}\right) p\right)$ is the Goette's infinitesimal equivariant eta invariant.

Proof. We still use the same notations and discussions after Proposition 3.8 in [Wa]. The difference is that we add $\psi_{t}$ in the definition of $A$. That is, let $A=d_{(u, s, t)}+\psi_{t} \widetilde{\mathbf{D}_{-} \mathbf{X}}$ be a superconnection on the trivial infinite dimensional superbundle with the base $[0,1] \times \mathbf{R} \times(0,+\infty)$ and the fibre $H \otimes \mathbf{C}^{\mathbf{r}} \oplus H \otimes \mathbf{C}^{\mathbf{r}}$. Then we have

$A^{2}=t \psi_{t} \mathbf{D}_{-X, u}^{2}-s^{2} / 4-(1-u) t^{\frac{1}{2}} s \sigma[\mathbf{D}, p]+d s \sigma\left(p-\frac{1}{2}\right)+t^{\frac{1}{2}} d u(2 p-1)[\mathbf{D}, p]+\frac{d t}{2 t^{\frac{1}{2}}} \psi_{t} \mathbf{D}_{X, u}$.

Since we prove the regularity at zero, we can take $\varepsilon=0$ in (3.41)-(3.45) in [Wa]. By the following lemma, Theorem 4.4 can be proved.

Lemma 4.5 Let $D_{u}=D+u(2 p-1)[D, p]$ for $u \in[0,1]$. We assume that $D$ be invertible and $\|d p\|<\lambda$, then we have $\eta_{X}\left(D_{0}\right)=\eta\left(D_{1}\right)$.

Proof. By $\|d p\|<\lambda$, then $D_{u}=D+u(2 p-1)[D, p]$ is invertible for $u \in[0,1]$. Similar to the discussions of Proposition 4.4 in [Wu], the infinitesimal equivariant eta invariant of $D_{u}$ is well defined. So $\eta_{X}\left(D_{u}\right)$ is smooth. Let $A=(2 p-1) d p$. Then by the definition of the infinitesimal equivariant eta invariant and the Duhamel principle, we have

$$
\frac{d}{d u} \eta_{X}\left(D_{u}\right)=\frac{1}{\sqrt{\pi}} \int_{0}^{+\infty} \operatorname{tr}\left[e^{-X} A e^{-t D_{-\frac{X}{t}, u}^{2}}\right] d \sqrt{t}+L
$$


where

$$
L=-\frac{t^{\frac{1}{2}}}{2 \sqrt{\pi}} \int_{0}^{+\infty} \int_{0}^{1} \operatorname{tr}\left\{e^{-X} D_{\frac{X}{t}, u} e^{-(1-s) t D_{-\frac{X}{t}, u}^{2}}\left[D_{-\frac{X}{t}, u}, A\right]_{+} e^{-s t D_{-\frac{X}{t}, u}^{2}} d s\right\} d t .
$$

By the trace property and direct computations, then

$$
\begin{aligned}
& \frac{\partial}{\partial t}\left(\sqrt{t} D_{u}+\frac{c(X)}{4 \sqrt{t}}\right)^{2}=\frac{1}{2}\left[D_{u}+\frac{c(X)}{4 t}, D_{u}-\frac{c(X)}{4 t}\right]_{+}, \\
& \int_{0}^{1} \operatorname{tr}\left\{A e^{-(1-s) t D_{-\frac{X}{t}, u}^{2}}\left[D_{-\frac{X}{t}, u}, D_{\frac{X}{t}, u}\right]_{+} e^{-s t D_{-\frac{X}{t}, u}^{2}}\right\} d s \\
& =\int_{0}^{1} \operatorname{tr}\left\{D_{\frac{X}{t}, u} e^{-(1-s) t D_{-\frac{X}{t}, u}^{2}}\left[D_{-\frac{X}{t}, u}, A\right]_{+} e^{-s t D_{-\frac{X}{t}, u}^{2}}\right\} d s .
\end{aligned}
$$

By using the Duhamel principle and the Leibniz rule and (4.26)-(4.28), we get

$$
\frac{\partial}{\partial u} \psi_{t} \operatorname{tr}\left[D_{X, u} e^{-t\left(D_{-X, u}^{2}+L_{X}\right)}\right] d \sqrt{t}=\frac{\partial}{\partial t} \operatorname{tr}\left[t^{\frac{1}{2}} e^{-X} A e^{-t D_{-\frac{X}{t}, u}^{2}}\right] d t .
$$

So

$$
\frac{d}{d u} \eta_{X}\left(D_{u}\right)=\left.\frac{1}{\sqrt{\pi}} \operatorname{tr}\left[t^{\frac{1}{2}} e^{-X} A e^{-t D^{2}}-\frac{X}{t}, u\right]\right|_{t=0} ^{+\infty} .
$$

As $D_{u}$ is invertible, then

$$
\lim _{t \rightarrow+\infty} \operatorname{tr}\left[t^{\frac{1}{2}} e^{-X} A e^{-t D_{-\frac{X}{t}, u}^{2}}\right]=0 .
$$

Using Lemma 2.9 in [Wa] for $j=n$ and $m=-1$, similar to the discussions on Line 14 in [Wu, P.164], we have

$$
\begin{gathered}
\lim _{t \rightarrow 0} \operatorname{tr}\left[t^{\frac{1}{2}} e^{-X} A e^{-t D^{2}-\frac{X}{t}, u}\right] \\
=c_{0} \int_{M} \widehat{A}\left(F_{\mathfrak{g}}^{M}(X)\right) \operatorname{tr}\left\{(2 p-1)(d p) \exp \left[\frac{\sqrt{-1}}{2 \pi}\left(A^{\prime} \wedge A^{\prime}+d A^{\prime}\right)\right]\right\}=0,
\end{gathered}
$$

where $A^{\prime}=u(2 p-1) d p$. Then by (4.30)-(4.32), Lemma 4.5 is proved.

Let $N$ be an even-dimensional compact manifold with the boundary $M$. We endow $N$ with a metric which is a product in a collar neighborhood of $M$. Denote by $D\left(D_{M}\right)$ the Dirac operator on $N(M)$. Let $C_{*}^{\infty}(N)=\left\{f \in C^{\infty}(N) \mid f\right.$ is independent of the normal coordinate $x_{n}$ near the boundary $\}$.

Definition 4.6 The infinitesimal equivariant Chern-Connes character on $N, \tau_{X}=$ $\left\{\tau_{X}^{0}, \tau_{X}^{2}, \cdots, \tau_{X}^{2 q} \cdots\right\}$ is defined by

$$
\tau_{X}^{2 q}\left(f^{0}, f^{1}, \cdot, f^{2 q}\right):=-\eta_{X}^{2 q}\left(D_{M}\right)\left(\left.f^{0}\right|_{M},\left.f^{1}\right|_{M}, \cdot,\left.f^{2 q}\right|_{M}\right)
$$




$$
+\frac{1}{(2 q) !(2 \pi \sqrt{-1})^{q}} \int_{M} \widehat{A}\left(F_{\mathfrak{g}}^{M}(X)\right) f^{0} d f^{1} \wedge \cdots \wedge d f^{2 q},
$$

where $f^{0}, f^{1}, \cdot, f^{2 q} \in C_{*}^{\infty}(N)$.

Similarly to Proposition 4.2 in [Wa1], we have

Proposition 4.7 The infinitesimal equivariant Chern-Connes character is $b-B$ closed (for the definitions of $b, B$, see [FGV]). That is, we have

$$
b \tau_{X}^{2 q-2}+B \tau_{X}^{2 q}=0
$$

By Proposition 4.3, we have

Proposition 4.8 Suppose that $D_{M}$ is invertible with $\lambda$ being the smallest positive eigenvalue of $\left|D_{M}\right|$. We assume that $\left\|d\left(\left.p\right|_{M}\right)\right\|<\lambda$, then the pairing $\left\langle\tau_{X}^{*}, \operatorname{ch}_{*}(p)\right\rangle$ is well-defined.

We let $C_{1}(M)=M \times(0,1], \tilde{N}=N \cup_{M \times\{1\}} C_{1}(M)$ and $\mathcal{U}$ be a collar neighborhood of $M$ in $N$. For $\varepsilon>0$, we take a metric $g^{\varepsilon}$ of $\widetilde{N}$ such that on $\mathcal{U} \cup_{M \times\{1\}} C_{1}(M)$

$$
g^{\varepsilon}=\frac{d r^{2}}{\varepsilon}+r^{2} g^{M}
$$

Let $S=S^{+} \oplus S^{-}$be spinors bundle associated to $\left(\widetilde{N}, g^{\varepsilon}\right)$ and $H^{\infty}$ be the set $\{\xi \in$ $\Gamma(\widetilde{N}, S) \mid \xi$ and its derivatives are zero near the vertex of cone $\}$. Denote by $L_{c}^{2}(\widetilde{N}, S)$ the $L^{2}$-completion of $H^{\infty}$ (similarly define $L_{c}^{2}\left(\widetilde{N}, S^{+}\right)$and $L_{c}^{2}\left(\widetilde{N}, S^{-}\right)$). Let

$$
D_{\varepsilon}: H^{\infty} \rightarrow H^{\infty} ; \quad D_{+, \varepsilon}: H_{+}^{\infty} \rightarrow H_{-}^{\infty},
$$

be the Dirac operators associated with $\left(\tilde{N}, g^{\varepsilon}\right)$ which are Fredholm operators for the sufficiently small $\varepsilon$. By $\left\|d\left(\left.p\right|_{M}\right)\right\|<\lambda$, then $p D_{M} p$ is invertible. Recall the Goette's infinitesimal equivariant index theorem for the twisting bundle $\operatorname{Im} p$ with the connection $p d$ in $[\mathrm{Go}]$ that

$$
\operatorname{Ind}_{e^{-X}}\left(p D_{+, \varepsilon} p\right)=\sum_{r=0}^{\infty} \frac{(-1)^{r}}{r !(2 \pi \sqrt{-1})^{r}} \int_{N} \widehat{A}\left(F_{\mathfrak{g}}^{N}(X)\right) \operatorname{Tr}\left[p(d p)^{2 r}\right]-\frac{1}{2} \eta_{X}\left(p D_{M} p\right) .
$$

By the Stokes theorem and the trace property and $p(d p)^{2}=(d p)^{2} p$, we have

$$
\int_{M} \widehat{A}\left(F_{\mathfrak{g}}^{M}(X)\right) \operatorname{tr}\left[p_{M}\left(d_{M} p_{M}\right)^{2 k-1}\right]=0
$$

By $L_{X}(p)=\iota_{X} d(p)=0$, then $\iota_{X}\left[p(d p)^{2 k-1}\right]=0$. By the Stokes theorem and (4.36), we get

$$
\int_{N} \widehat{A}\left(F_{\mathfrak{g}}^{N}(X)\right) \operatorname{tr}\left[\left(d_{N} p_{N}\right)^{2 k}\right]=\int_{N}\left(d+\iota_{X}\right)\left[\widehat{A}\left(F_{\mathfrak{g}}^{N}(X)\right) \operatorname{tr}\left[p\left(d_{N} p_{N}\right)^{2 k-1}\right]\right]
$$




$$
=\int_{M} \widehat{A}\left(F_{\mathfrak{g}}^{M}(X)\right) \operatorname{tr}\left[p_{M}\left(d_{M} p_{M}\right)^{2 k-1}\right]=0 .
$$

By Theorem 4.4 and Definition 4.6 and (2.14) and (4.37), we get

Theorem 4.9 Suppose that $D_{M}$ is invertible with $\lambda$ being the smallest positive eigenvalue of $\left|D_{M}\right|$. We assume that $\left\|d\left(\left.p\right|_{M}\right)\right\|<\lambda$ and $p \in M_{r \times r}\left(C_{*}^{\infty}(N)\right)$, then

$$
\operatorname{Ind}_{e^{-X}}\left(p D_{+, \varepsilon} p\right)=\left\langle\tau_{X}^{*}(D), \operatorname{ch}_{*}(p)\right\rangle .
$$

\section{The infinitesimal equivariant Chern-Connes character for a family of Dirac operators}

In this Section, we extend Sections 2, 3 to the family case. Let us recall the definition of the equivariant family Bismut Laplacian. Let $M$ be a $n+q$ dimensional compact connected manifold and $B_{0}$ be a $q$ dimensional compact connected manifold. Assume that $\pi: M \rightarrow B_{0}$ is a fibration and $M$ and $B_{0}$ are oriented. Taking the orthogonal bundle of the vertical bundle $T Z$ in $T M$ with respect to any Riemannian metric, determines a smooth horizontal subbundle $T^{H} M$, i.e. $T M=T^{H} M \oplus T Z$. Recall that $B_{0}$ is Riemannian, so we can lift the Euclidean scalar product $g_{B_{0}}$ of $T B_{0}$ to $T^{H} M$. And we assume that $T Z$ is endowed with a scalar product $g_{Z}$. Thus we can introduce a new scalar product $g_{B_{0}} \oplus g_{Z}$ in $T M$. Denote by $\nabla^{L}$ the LeviCivita connection on $T M$ with respect to this metric. Let $\nabla^{B_{0}}$ denote the Levi-Civita connection on $T B_{0}$ and still denote by $\nabla^{B_{0}}$ the pullback connection on $T^{H} M$. Let $\nabla^{Z}=P_{Z}\left(\nabla^{L}\right)$, where $P_{Z}$ denotes the projection to $T Z$. Let $\nabla^{\oplus}=\nabla^{B_{0}} \oplus \nabla^{Z}$ and $\omega=\nabla^{L}-\nabla^{\oplus}$ and $T$ be the torsion tensor of $\nabla^{\oplus}$. Now we assume that the bundle $T Z$ is spin. Let $S(T Z)$ be the associated spinors bundle and $\nabla^{Z}$ can be lifted to give a connection on $S(T Z)$. Let $D$ be the tangent Dirac operator.

Let $G$ be a compact Lie group which acts fiberwise on $M$. We will consider that $G$ acts as identity on $B_{0}$. We assume that the action of $\mathrm{G}$ lifts to $S(T Z)$ and the $G$-action commutes with $D$. Let $E$ be the vector bundle $\pi^{*}\left(\wedge T^{*} B_{0}\right) \otimes S(T Z)$. This bundle carries a natural action $m_{0}$ of the degenerate Clifford module $C l_{0}(M)$. Define the connection for $X \in \mathfrak{g}$ whose Killing vector field is in $T Z$,

$$
\begin{aligned}
\nabla^{E,-X, \oplus} & :=\pi^{*} \nabla^{B_{0}} \otimes 1+1 \otimes \nabla^{S,-X}, \\
\omega(Y)(U, V) & :=g\left(\nabla_{Y}^{L} U, V\right)-g\left(\nabla_{Y}^{\oplus} U, V\right), \\
\nabla_{Y}^{E,-X, 0} & :=\nabla_{Y}^{E,-X, \oplus}+\frac{1}{2} m_{0}(\omega(Y)),
\end{aligned}
$$

for $Y, U, V \in T M$. Then the equivariant Bismut superconnection acting on $\Gamma\left(M, \pi^{*} \wedge\right.$ $\left.\left(T^{*} B_{0}\right) \otimes S(T Z)\right)$ is defined by

$$
B^{-X}=\sum_{i=1}^{n} c\left(e_{i}^{*}\right) \nabla_{e_{i}}^{E,-X, 0}+\sum_{j=1}^{q} f_{j}^{*} \wedge \nabla_{f_{j}}^{E,-X, 0} ; \quad B^{-X}=B+\frac{1}{4} c(X) .
$$


where $e_{1}, \cdots, e_{n}$ and $f_{1}, \cdots, f_{q}$ are orthonormal basis of $T Z$ and $T B_{0}$ respectively, and $B$ is the Bismut superconnection defined by

$$
\begin{gathered}
\nabla^{E, \oplus}:=\pi^{*} \nabla^{B_{0}} \otimes 1+1 \otimes \nabla^{S} ; \\
\nabla_{Y}^{E, 0}:=\nabla_{Y}^{E, \oplus}+\frac{1}{2} m_{0}(\omega(Y)) ; \\
B=\sum_{i=1}^{n} c\left(e_{i}^{*}\right) \nabla_{e_{i}}^{E, 0}+\sum_{j=1}^{\bar{q}} c\left(f_{j}^{*}\right) \nabla_{f_{j}}^{E, 0} .
\end{gathered}
$$

Define the equivariant family Bismut Laplacain as follows:

$$
H_{B, X}=\left(B^{-X}\right)^{2}+L_{X}^{E}
$$

where $L_{X}^{E}$ is the Lie derivative induced by $X$ on the bundle $E$. Then

$$
H_{B, X}=D^{2}+F_{+}+\widetilde{F}_{+},
$$

where $D_{-X}^{2}=D^{2}+F_{+}$and $\widetilde{F}_{+}=H_{B, X}-D_{-X}^{2}$ is a first order differential operator along the fibre with coefficients in $\Omega_{\geq 1}\left(B_{0}\right)$.

Definition 5.1 The infinitesimal equivariant family JLO $\operatorname{cochain} \operatorname{ch}^{2 k}(B, X)$ can be defined by the formula for $f^{0}, \cdots, f^{2 k}$ in $C_{G}^{\infty}(M)$ :

$$
\begin{gathered}
\operatorname{ch}^{2 k}(B, X)\left(f^{0}, \cdots, f^{2 k}\right):=\int_{\triangle_{2 k}} \operatorname{Str}\left[f^{0} e^{-\sigma_{0} H_{B, X}} c\left(d f^{1}\right) e^{-\sigma_{1} H_{B, X}}\right. \\
\left.\cdots c\left(d f^{2 k}\right) e^{-\sigma_{2 k} H_{B, X}}\right] d \mathrm{Vol}_{\Delta_{2 k}},
\end{gathered}
$$

where Str is taking the trace along the fibre.

Similarly to Section 2 , we can prove that (5.10) is well-defined and $\left\langle\operatorname{ch}^{*}(B, X), \operatorname{ch} p\right\rangle$ is convergent by the following lemma.

Lemma 5.2 For any $1 \geq u>0$, we have:

$$
\left\|e^{-u H_{B, X}}\right\|_{u^{-1}} \leq C_{0} e^{\left\|F_{X}\left(1+D^{2}\right)^{-\frac{1}{2}}\right\| \pi u}\left(\operatorname{tr}\left[e^{-\frac{D^{2}}{2}}\right]\right)^{u},
$$

where the constant $C_{0}$ is independent of $u$.

Proof. By (5.10) and the Duhamel principle, we have

$$
e^{-u H_{B, X}}=e^{-u H_{X}}+\sum_{r>0}^{\operatorname{dim} B_{0}} I_{r},
$$

where

$$
I_{r}=\int_{\triangle_{r}} e^{-s_{0} u H_{X}} \widetilde{F}_{+} e^{-s_{1} u H_{X}} \cdots \widetilde{F}_{+} e^{-s_{r} u H_{X}} d s
$$


In (4.1), we use $\widetilde{F}_{+}$and $s u$ instead of $D_{-X}$ and $u$ respectively and let $t=1$, then we have

$$
\begin{gathered}
\left\|\widetilde{F}_{+} e^{-s u H_{X}}\right\|_{(s u)^{-1}} \leq 2(s u)^{-\frac{1}{2}}\left\|\widetilde{F}_{+}\left(1+D^{2}\right)^{-\frac{1}{2}}\right\| e^{\frac{s u}{2}} \\
\cdot\left\{1+\left[\left\|F_{X}\left(1+D^{2}\right)^{-\frac{1}{2}}\right\|^{2} e^{-1} \pi s u\right]^{\frac{1}{2}}\right\} e^{\left\|F_{X}\left(1+D^{2}\right)^{-\frac{1}{2}}\right\|^{2} \pi s u}\left(\operatorname{tr} e^{-\frac{1}{2} D^{2}}\right)^{s u} .
\end{gathered}
$$

By Lemma 2.2 and (5.12)-(5.14) and the Hölder inequality, we get Lemma 5.2.

Similarly to Propositions 4.11 and 4.12 in $[\mathrm{BC}]$, we have

Proposition 5.3 (1) The infinitesimal equivariant family Chern-Connes character is closed:

$$
\left(B+b+d_{B_{0}}\right)\left(\operatorname{ch}^{*}(B, X)\right)=0 .
$$

(2) Let $B_{\tau}=B^{-X}+\tau V$ and $V$ is a bounded operator which commutes with $e^{-X}$, then there exists a cochain $\operatorname{ch}^{*}\left(B_{\tau}, X, V\right)$ such that

$$
\frac{d}{d \tau} \operatorname{ch}^{*}\left(B_{\tau}, X\right)=-\left[b+B+d_{B_{0}}\right] \operatorname{ch}^{*}\left(B_{\tau}, X, V\right) .
$$

By taking $V=(2 p-1)[B, p]$, we get

Theorem 5.4 The following index formula holds in the cohomology of $B_{0}$

$$
\mathrm{Ch}_{e^{-X}}\left[\operatorname{Ind}\left(D_{\operatorname{Im} p,+, z}\right)\right]=\left\langle\operatorname{ch}^{*}(B, X), \operatorname{ch}(p)\right\rangle .
$$

Let $\phi_{t}$ be the rescaling operator on $\Omega\left(B_{0}\right)$ defined by $d y_{j} \rightarrow \frac{d y_{j}}{\sqrt{t}}$ for $t>0$. By the method in Section 4 in [Wa], similarly to Theorem 2.12 in [Wa], we get

Lemma 5.5 When $2 k \leq n$ and $X$ is small, then for $f^{j} \in C_{G}^{\infty}(M)$,

$$
\begin{gathered}
\lim _{t \rightarrow 0} \phi_{t} \psi_{t} \operatorname{ch}^{2 k}(\sqrt{t} B, X)\left(f^{0}, \cdots, f^{2 k}\right)_{J} \\
=\frac{1}{(2 k) !}(2 \pi \sqrt{-1})^{-n / 2} \int_{Z} f^{0} \wedge d f^{1} \wedge \cdots \wedge d f^{2 k} \widehat{A}\left(F_{\mathfrak{g}}^{Z}(X)\right)_{J} .
\end{gathered}
$$

Extending Theorem 3.9 to the family case, we have by Lemma 5.5 by

Theorem 5.6 When $2 k \leq n$ and $X$ is small, then for $f^{j} \in C_{G}^{\infty}(M)$,

$$
\begin{gathered}
\lim _{t \rightarrow 0} \phi_{t} \psi_{t} \operatorname{ch}^{2 k}(\sqrt{t} B, X)\left(f^{0}, \cdots, f^{2 k}\right) \\
=\frac{1}{(2 k) !}(2 \pi \sqrt{-1})^{-n / 2} \int_{Z} f^{0} \wedge d f^{1} \wedge \cdots \wedge d f^{2 k} \widehat{A}\left(F_{\mathfrak{g}}^{Z}(X)\right) .
\end{gathered}
$$


By Theorems 5.4 and 5.6 and the following homotopy property, similarly to Corollary 3.11 , we have

Corollary 5.7 When $X$ is small, then

$$
\mathrm{Ch}_{e^{-X}}\left[\operatorname{Ind}\left(D_{\operatorname{Im} p,+, z}\right)\right]=(2 \pi \sqrt{-1})^{-n / 2} \int_{Z} \widehat{A}\left(F_{\mathfrak{g}}^{Z}(X)\right) \operatorname{Ch}(\operatorname{Im} p) .
$$

Let $B_{t}=\sqrt{t} \phi_{t} \psi_{t}\left(B^{-X}\right)$ and $\mathcal{F}_{t}=B_{t}^{2}$. Then we have the homotopy formula:

Proposition 5.8 There is a cochain $\operatorname{ch}\left(B_{t}, \frac{d B_{t}}{d t}, X\right)$ such that the following formula holds

$$
\frac{d \operatorname{ch}\left(B_{t}, X\right)}{d t}=-\left(b+B+d_{B_{0}}\right) \operatorname{ch}\left(B_{t}, \frac{d B_{t}}{d t}, X\right) .
$$

Proof. We know that $B_{t}$ is a superconnection on the infinite dimensional bundle $C^{\infty}(M, E) \rightarrow B_{0}$ which we write $\mathcal{E} \rightarrow B_{0}$. Let $\widetilde{B_{0}}=B_{0} \times \mathbb{R}_{+}$, and $\widetilde{\mathcal{E}}$ be the superbundle $\pi^{*} \mathcal{E}$ over $\widetilde{B_{0}}$, which is the pull-back to $\widetilde{B_{0}}$ of $\mathcal{E}$. Define a superconnection $\widehat{B}$ on $\widetilde{\mathcal{E}}$ by the formula

$$
(\widehat{B} \beta)(x, t)=\left(B_{t} \beta(\cdot, t)\right)(x)+d t \wedge \frac{\partial \beta(x, t)}{\partial t} .
$$

The curvature $\widehat{\mathcal{F}}$ of $\widehat{B}$ is

$$
\widehat{\mathcal{F}}=\mathcal{F}_{t}-\frac{d B_{t}}{d t} \wedge d t
$$

where $\mathcal{F}_{t}=B_{t}^{2}$ is the curvature of $B_{t}$. By the Duhamel principle, then

$$
e^{-\widehat{\mathcal{F}}}=e^{-\mathcal{F}_{t}}+\left(\int_{0}^{1} e^{-u \mathcal{F}_{t}} \frac{d B_{t}}{d t} e^{-(1-u) \mathcal{F}_{t}} d u\right) \wedge d t .
$$

Let $f^{0}, \cdots, f^{2 k}$ be in $C_{G}^{\infty}(M)$, then $\left[\widehat{B}, f^{j}\right]=\left[B_{t}, f^{j}\right]$. We replace $K_{t}$ in (4.5) and (4.6) by the above $B_{t}$, then we define the cochain $\operatorname{ch}\left(B_{t}, \frac{d B_{t}}{d t}, X\right)$. So by (5.24), we get on $C_{G}^{\infty}(M)$ that

$$
\operatorname{ch}(\widehat{B}, X)=\operatorname{ch}\left(B_{t}, X\right)+\operatorname{ch}\left(B_{t}, \frac{d B_{t}}{d t}, X\right) d t
$$

Similarly to (5.15), we have

$$
\left(b+B+d_{\widetilde{B_{0}}}\right) \operatorname{ch}(\widehat{B}, X)=0 ; \quad\left(b+B+d_{B_{0}}\right) \operatorname{ch}\left(B_{t}, X\right)=0 .
$$

By (5.25) and (5.26), we get Proposition 5.8.

Acknowledgements. This work was supported by NSFC No.11271062 and NCET13-0721. The author would like to thank Profs. Weiping Zhang and Huitao Feng for 
very helpful suggestions and discussions. The author would like to thank the referee for careful reading and helpful comments.

\section{References}

[ABP $]$ M. Atiyah, R. Bott, V. Patodi, On the heat equation and the index theorem, Invent. Math., 19 (1973), 279-330.

[Az]F. Azmi, The equivariant Dirac cyclic cocycle, Rocky Mountain J. Math. 30(2000), 1171-1206.

[BC]M. Benameur, A. Carey, Higher spectral flow and an entire bivariant JLO cocycle, J. K-theory, 11 (2013), 183-232.

[BGV]N. Berline, E. Getzler and M. Vergne, Heat kernels and Dirac operators, SpringerVerlag, Berlin, 1992.

[BV]N. Berline and M. Vergne, The equivariant index and Kirillov character formula, Amer. J. Math. 107 (1985), 1159-1190.

[BV1]N. Berline and M. Vergne, A computation of the equivariant index of the Dirac operators, Bull. Soc. Math. France 113 (1985), 305-345.

[Bi]J. M. Bismut, The infinitesimal Lefschetz formulas: a heat equation proof, J. Func. Anal. 62 (1985), 435-457.

[BiF] J. M. Bismut and D. S. Freed, The analysis of elliptic families II, Commun. Math. Phys. 107 (1986), 103-163.

$[\mathrm{BF}] J$. Block and J. Fox, Asymptotic pseudodifferential operators and index theory, Contemp. Math., 105 (1990), 1-32.

$[\mathrm{CH}] \mathrm{S}$. Chern and X. Hu, Equivariant Chern character for the invariant Dirac operators, Michigan Math. J. 44 (1997), 451-473.

[Co]A. Connes, Entire cyclic cohomology of Banach algebras and characters of $\theta$ summable Fredholm module, K-Theory 1 (1988), 519-548.

$[\mathrm{CM}] \mathrm{A}$. Connes and H. Moscovici, Cyclic cohomology, the Novikov conjecture and hyperbolic groups, Topology 29 (1990), 345-388.

[Do]H. Donnelly, Eta invariants for G-space, Indiana Univ. Math. J. 27 (1978), 889-918.

[Fe]H. Feng, A note on the noncommutative Chern character (in Chinese), Acta Math. Sinica 46 (2003), 57-64.

[FGV]H. Figueroa, J. Gracia-Bondía and J. Várilly, Elements of noncommutative geometry, Birkhäuser Boston, 2001.

[Ge1]E. Getzler, The odd Chern character in cyclic homology and spectral flow, Topology 32 (1993), 489-507.

[Ge2]E. Getzler, Pseudodifferential operators on supermanifolds and the AtiyahSinger index theorem, Comm. Math. Phys. 92 (1983), no. 2, 163-178.

[GS]E. Getzler and A. Szenes, On the Chern character of theta-summable Fredholm modules, J. Func. Anal. 84 (1989), 343-357.

[Go]S. Goette, Equivariant eta invariants and eta forms, J. reine angew Math. 526 (2000), 181-236. 
[JLO]A. Jaffe, A. Lesniewski and K. Osterwalder, Quantum K-theory: The Chern character, Comm. Math. Phys. 118 (1988), 1-14.

[KL]S. Klimek and A. Lesniewski, Chern character in equivariant entire cyclic cohomology, K-Theory 4 (1991), 219-226.

[LYZ]J. D. Lafferty, Y. L. Yu and W. P. Zhang, A direct geometric proof of Lefschetz fixed point formulas, Trans. AMS. 329 (1992), 571-583.

[Pf]M. Pflaum, The normal symbol on Riemannian manifolds. New York J. Math. 4 (1998), 97-125.

[PW]R. Ponge and H. Wang, Noncommutative Geometry and Conformal Geometry. II. Connes-Chern character and the local equivariant index theorem, arXiv:1411.3703.

[Si]B. Simon, Trace ideals and their applications, London Math. Soc., Lecture Note 35, 1979.

[Wa]Y. Wang, The noncommutative infinitesimal equivariant index formula, J. KTheory, 14 (2014), 73-102.

[Wa1]Y. Wang, The equivariant noncommutative Atiyah-Patodi-Singer index theorem, K-Theory 37 (2006), 213-233.

[Wu] F. Wu, The Chern-Connes character for the Dirac operators on manifolds with boundary, K-Theory 7 (1993), 145-174.

[Zh]W. P. Zhang, A note on equivariant eta invariants, Proc. AMS. 108 (1990), 11211129.

School of Mathematics and Statistics, Northeast Normal University, Changchun Jilin, 130024, China

E-mail: wangy581@nenu.edu.cn 